\title{
Microsurgical epididymal sperm aspiration: indications, techniques and outcomes
}

\author{
Aaron M Bernie ${ }^{1}$, Ranjith Ramasamy ${ }^{1}$, Doron S Stember ${ }^{2}$ and Peter J Stahl ${ }^{3}$ \\ Microsurgical epididymal sperm aspiration (MESA) refers to retrieval of sperm-containing fluid from optimal areas of the epididymis \\ that are selected and sampled using high-power optical magnification provided by an operating microscope. Retrieved sperm are \\ subsequently used for intracytoplasmic sperm injection (ICSI) to induce fertilization and pregnancy. MESA is considered by many \\ experts to be the gold standard technique for sperm retrieval in men with obstructive azoospermia given its high yield of quality sperm, \\ excellent reported fertilization and pregnancy rates, and low risk of complications. However, MESA must be performed in an operating \\ room, requires microsurgical skills and is only useful for reproduction using ICSI. Herein we present an overview of the evaluation of \\ candidate patients for MESA, the technical performance of the procedure and the outcomes that have been reported. \\ Asian Journal of Andrology (2013) 15, 40-43; doi:10.1038/aja.2012.114; published online 19 November 2012
}

Keywords: azoospermia; ICSI; infertility; male; MESA; microsurgical epididymal sperm aspiration; microsurgery; sperm retrieval

\section{INTRODUCTION}

Technical refinements in sperm retrieval methods and the application of intracytoplasmic sperm injection (ICSI) have enabled biological paternity in azoospermic men who were considered untreatable 20 years ago. Achievement of optimal reproductive outcomes in such patients depends upon selection of the optimal sperm acquisition procedure for each individual patient, and upon the proper technical performance of the selected procedure. For men with obstructive azoospermia (OA), microsurgical epididymal sperm aspiration (MESA) is considered by most andrologists to be the gold standard procedure for sperm acquisition. During MESA, sperm-containing epididymal fluid is aspirated from optimal areas of the epididymis that are selected and sampled using high-power optical magnification provided by an operating microscope. Herein we review the evaluation of candidate patients for MESA, important technical points for performance of the procedure and the outcomes of MESA that have been reported.

\section{CLINICAL EVALUATION OF CANDIDATES FOR MESA}

The clinical evaluation of azoospermic men who are candidates for MESA should focus upon assessing the couple's reproductive goals, confirming that the azoospermia is obstructive in etiology, and identifying medically important or genetically transmissible disease (cystic fibrosis). MESA is not indicated in cases of non-obstructive azoospermia (NOA). Sperm are unlikely to be found in the epididymis when sperm production is severely impaired, and puncture of a non-dilated epididymal tubule is extremely technically challenging and unlikely to be successful.

The reproductive goals of the couple play an important role in selection of the optimal surgical treatment for azoospermia and should be specifically discussed. Each couple should be asked about their feelings and beliefs about the use of assisted reproduction. Patients with objections to assisted reproduction may be better served by microsurgical reconstruction to bypass obstruction (when clinically appropriate) than by surgical sperm retrieval for ICSI. The number of desired children is another important point for discussion, as the various options for sperm acquisition vary significantly in the quantity of sperm retrieved and the likelihood of retrieving enough sperm for cryopreservation and repeated cycles of assisted reproduction. In general, percutaneous sperm retrieval procedures usually do not yield enough sperm for multiple cycles.

The history is important for identification of factors that may predispose to OA and NOA. ${ }^{1}$ Patients should be specifically asked about risk factors for NOA including delayed or incomplete pubertal development, history of cryptorchidism and prior gonadotoxin exposures (such as chemotherapy or radiation for cancer treatment). Inquiry should also be specifically directed at identification of risk factors for OA including prior vasectomy, prior inguinal or scrotal surgery and history of epididymitis or sexually transmitted infections. The patient's prior reproductive history is critical, with a prior history of biological paternity being strongly suggestive of OA (unless exposures that threaten fertility occurred subsequent to prior reproduction). Patients should also be asked about chronic brochopulmonary infections or gastrointestinal disorders, which may be associated with congenital bilateral absence of the vasa deferentia (CBAVD), a common cause of OA. ${ }^{1}$

Physical examination should focus upon determination of the volume, consistency and presence of each testis, epididymis and vas deferens. Small soft testes suggest impaired sperm production, while indurated epididymities or absence of palpable vasa deferentia with

${ }^{1}$ Weill Cornell Medical College, New York-Presbyterian Hospital, New York, NY 10021, USA; ${ }^{2}$ Beth Israel Medical Center of Albert Einstein College of Medicine of Yeshiva University, New York, NY 10003, USA and ${ }^{3}$ Columbia University College of Physicians \& Surgeons, New York-Presbyterian Hospital, New York, NY 10032, USA Correspondence: Dr PJ Stahl (ps2192@columbia.edu)

Received: 10 September 2012; Revised: 3 October 2012; Accepted: 3 October 2012; Published online: 19 November 2012 
normal volume testes suggest obstruction. ${ }^{2}$ Digital rectal examination of the prostate should be performed to assess for seminal vesicle dilation and palpable midline prostate abnormalities, which suggest ejaculatory duct obstruction.

Semen analysis is critical for confirmation of azoospermia by the absence of any sperm in the pellet derived from semen centrifugation. Occasionally rare sperm may be found in the ejaculate that may be used for ICSI and obviate the need for retrieval. Moreover, the gross semen characteristics provide important clues about the nature of azoospermia. The seminal vesicles produce alkaline, fructose-positive fluid that comprises the majority $(50 \%-80 \%)$ of the ejaculate volume. Therefore, low volume $(<1.5 \mathrm{ml})$, acidic $(\mathrm{pH}<7.0)$, fructose-negative azoospermia suggests absence of the seminal vesicle contribution to the ejaculate. This finding implies either obstruction of the ejaculatory ducts or malformation of the seminal vesicles, which is often associated with CBAVD. In contrast, normal volume, normal $\mathrm{pH}$, fructosepositive azoospermia suggests either epididymal or vasal obstruction or NOA. $^{3}$

Laboratory testing should include serum tests for testosterone (T) and follicle-stimulating hormone (FSH). ${ }^{4}$ Normal serum hormone levels suggest $\mathrm{OA}$, particularly in the setting of normal testicular volumes. Although individual laboratory normal ranges are highly variable, young healthy males with normal sperm production should have a serum FSH less than $7.6 \mathrm{IU} \mathrm{l}^{-1}$ and an early morning serum total $\mathrm{T}$ greater than $300 \mathrm{ng} \mathrm{dl}{ }^{-1}$. The value in routinely obtaining serum $\mathrm{T}$ and FSH levels in all azoospermic patients considering a sperm acquisition procedure, even when there is an obvious cause for obstruction, was nicely delineated by Meng et al. ${ }^{5}$ This group evaluated sperm production in 33 men with CBAVD (who would usually be assumed to have OA) and found that $4 / 33$ (12\%) patients had cytological or histological evidence of impaired spermatogenesis. The patients with normal sperm production had larger testicular volumes and statistically lower serum FSH levels compared to those men with abnormal spermatogenesis (mean FSH 4.9 vs. $9.3 \mathrm{IU}^{-1}$, respectively; $P=0.012$ ).

Patients with CBAVD on physical examination or unexplained OA with low semen volume should be tested for abnormalities of the cystic fibrosis transmembrane conductance regulator gene, which encodes a transmembrane chloride channel that plays a critical role in the embryological development of the seminal vesicles, vasa deferentia and epididymis. Fifty to eighty-two percent of such men have detectable mutations in one or both cystic fibrosis transmembrane conductance regulator alleles. ${ }^{6}$ Testing of the female partner is also crucial, even when the male tests negative, because most clinically available screening panels test for only $30-50$ of the roughly 1900 mutations that have been described. Cystic fibrosis transmembrane conductance regulator testing is critical in appropriately selected azoospermic patients for preconception genetic counseling, as these couples are at risk of having offspring that may have overt cystic fibrosis or infertility.

\section{SELECTION OF THE OPTIMAL SPERM RETRIEVAL PROCEDURE: MESA AND ALTERNATIVES}

OA is characterized by diffuse distribution of normal or near-normal sperm production throughout the testes. Millions of sperm accumulate in the dilated portions of the vas deferens and epididymis on the testicular side of the anatomic point of obstruction. In most cases, motile sperm may be found in the proximal portions of the epididymis and even in the testes of men with OA. ${ }^{7}$ Given the wide distribution of sperm throughout the testes and epididymities in men with OA, sperm retrieval is possible in most cases using any percutaneous or surgical technique. However, each sperm acquisition procedure is associated with different anesthetic requirements, safety profiles, required surgical skills and sperm yields. MESA, percutaneous epididymal sperm aspiration, fine and large needle aspiration of sperm from the testes, conventional testicular sperm extraction and microdissection testicular sperm extraction have all been successfully utilized in patients with $\mathrm{OA}$. The performance characteristics of each sperm acquisition procedure are presented in Table $1 .^{8-20}$ MESA is the gold standard approach because it yields millions of high-quality, motile sperm with minimal specimen contamination with red blood cells. ${ }^{12}$

There is controversy about whether or not the anatomic source of sperm used for ICSI cycles impacts reproductive outcomes. A 2004 meta-analysis did not find any differences in fertilization rates, clinical pregnancy rates or live births rates when comparing outcomes of ICSI cycles using epididymal and testicular sperm. ${ }^{21}$ Moreover, a 2008 Cochrane review failed to identify significant differences in ICSI outcomes when comparing testicular and epididymal sperm, and the authors concluded that it was not possible to recommend one method of sperm acquisition over any other for azoospermic men. It is important to note, however, that the Cochrane recommendations were based predominantly on two small trials that showed no differences in assisted reproduction outcomes (one study compared MESA to percutaneous epididymal sperm aspiration and the other compared testicular sperm aspiration both with and without ultrasound guidance) ${ }^{22}$

\section{Table 1 Performance characteristics of sperm acquisition procedures for men with obstructive azoospermia ${ }^{8-20}$}

\begin{tabular}{|c|c|c|c|}
\hline Procedure & Anesthetic & Sperm retrieval rate & Yield \\
\hline MESA & General (preferred), regional & $95 \%-100 \%$ & $\begin{array}{c}\text { Average of } 15-95 \times 10^{6} \text { total sperm with } 15 \%-42 \% \text { total motility, cryopreservation } \\
\text { possible in } 98 \%-100 \% \text { of cases with an average of } 5.3-7.6 \text { vials per patient }\end{array}$ \\
\hline PESA & Local & $80 \%-100 \%$ & $\begin{array}{l}\text { Thousands to millions of sperm with variable motility (poorly reported in most } \\
\text { studies), cryopreservation possible in } 43 \%-96 \% \text { of cases }\end{array}$ \\
\hline Testicular fine needle aspiration & Local & $52 \%-100 \%$ & $\begin{array}{l}\text { Hundreds of thousands to millions of sperm with variable motility (poorly reported } \\
\text { in most studies), cryopreservation possible in } 38 \% \text { of cases in one study }\end{array}$ \\
\hline Testicular large needle aspiration & Local & $98 \%-100 \%$ & $\begin{array}{l}\text { Hundreds of thousands to millions of sperm with variable motility (poorly reported } \\
\text { in most studies), cryopreservation possible in } 100 \% \text { of cases in one study }\end{array}$ \\
\hline Testicular core needle biopsy & Local & $82 \%-100 \%$ & $\begin{array}{l}\text { Hundreds of thousands to millions of sperm with variable motility (poorly reported } \\
\text { in most studies), often sufficient for cryopreservation (poorly reported) }\end{array}$ \\
\hline TESE & General, regional, local & $100 \%$ & $\begin{array}{l}\text { Hundreds of thousands to millions of sperm in most cases (poorly reported in most } \\
\text { studies), usually sufficient for cryopreservation (poorly reported) }\end{array}$ \\
\hline Microdissection TESE & General (preferred), regional & $100 \%$ & $\begin{array}{l}\text { Hundreds of thousands to millions of sperm in most cases (poorly reported in most } \\
\text { studies), usually sufficient for cryopreservation (poorly reported) }\end{array}$ \\
\hline
\end{tabular}


However, there are retrospective and single-center studies that have suggested that ICSI outcomes may be better using epididymal sperm. One study of 368 ICSI cycles in 171 men with OA showed a significantly higher rate of pregnancy loss when testicular sperm were used, suggesting that it may be preferential to use epididymal sperm for assisted reproduction in men with OA. ${ }^{23}$ Moreover, one single-center study reported a $100 \%$ per couple live delivery rate in patients with OA due to CBAVD who were treated by MESA, emphasizing the excellent efficacy and potential superiority of this approach in properly selected patients. ${ }^{18,24}$

Another important and relevant question that has been raised is whether or not ICSI outcomes vary based on the use of fresh $v s$. frozen epididymal sperm samples. A study by Cayan et al. ${ }^{25}$ suggested that cryopreserved and thawed sperm retrieved from the epididymis produce similar success rates when used for ICSI. They found no significant difference in fertilization rate $58.4 \%$ for fresh sperm and $62 \%$ for frozen-thawed sperm), clinical pregnancy rates (31.6\% for fresh sperm and $36.8 \%$ for frozen-thawed sperm) or live-birth rate $(21.1 \%$ for fresh sperm and $36.8 \%$ for frozen-thawed sperm) ${ }^{25}$ Others have confirmed that the viability of fresh and frozen-thawed retrieved sperm is comparable when the proper methods are used to assess sperm viability. Bachtell et al. ${ }^{26}$ showed that fresh sperm retrieved from the vas deferens, testis and epididymis exhibit similar viability to sperm from the same sites that are frozen-thawed.

\section{MESA: TECHNICAL PERFORMANCE}

General anesthesia, spinal or local anesthesia may be used. General anesthesia is generally preferred to minimize patient movement during the procedure, which is highly accentuated by the high-power optical magnification that is utilized. Suggested equipment includes a $\times 6-\times 30$ operating microscope, surgical and microsurgical instruments including an ophthalmic microknife, a bench-top phase contrast or light microscope, $5 \mu \mathrm{l}$ glass pipettes for collection of epididymal fluid, silicone tubing that attaches to both a syringe and the pipettes, human tubule fluid (HTF) or another appropriate sperm transport solution, and glass slides with cover slips. ${ }^{27}$

After induction of general anesthesia, the patient's scrotum is shaved, prepped and draped in sterile fashion. The scrotal skin, dartos muscle and tunica vaginalis are divided to expose the testis and epididymis. The surgeon grasps the testis with his or her non-dominant hand and stabilizes the epididymis between his or her thumb and index finger. The epididymis is inspected at $\times 10-\times 15$ magnification to select dilated tubules containing golden, semitranslucent fluid (usually in the caput) for aspiration. Opaque, yellow tubules typically contain only sperm heads and debris and should be avoided.

The epididymal tubules are exposed by careful incision or excision of the overlying epididymal tunic at $\times 15-\times 20$ magnification. Meticulous hemostasis is obtained with bipolar electrocautery to avoid contamination of the epididymal fluid with blood, avoiding the application of excess heat to the underlying epididymal tubules. The epididymal tubule can then be sharply punctured with an ophthalmic microknife. At this point, real-time analysis of sperm quantity and quality within the epididymal fluid should be performed to direct the duration and extent of the procedure (discussed later).

Continued epididymal exploration and repeat epididymal punctures should be performed until an epididymal site containing microscopically adequate fluid is identified, moving toward the caput of the epididymis with each successive puncture that does not yield sufficient quality epididymal fluid. In nearly all patients, better sperm motility is found closer to the testis in the caput region of the epididymis. It is exceedingly rare to have no sperm motility in all areas of the obstructed epididymis. Such a finding should alert the surgeon that they are too far distal in the obstructed reproductive tract, except in the extremely rare patient with ciliary dysfunction. If necessary, the efferent ductules may be exposed by reflection of the caput epididymis away from the testis and individual tubules are aspirated with compression of the testis to facilitate outflow of intratesticular fluid. Fluid retrieved from the efferent ductules has been shown to exhibit similar sperm concentration and motility to that of sperm retrieved from the proper epididymis, although it should be noted that the sperm from the efferent ductules may be less durable when exposed to cryopreservation and thawing. ICSI outcomes also appear to be similar using sperm derived from the efferent ductules and proper epididymis. ${ }^{28}$

When adequate epididymal fluid is identified, it is collected atraumatically with glass micropipettes by simple capillary action. The tip of a 5 - $\mu$ l micropipette $(0.5 \mathrm{~mm}$ luminal diameter and $0.9 \mathrm{~mm}$ outer diameter) is placed adjacent to the effluxing epididymal tubule (Figure 1). Fluid is collected into multiple pipettes until its flow ceases or enough high-quality epididymal fluid has been collected. Ten to 20 $\mu \mathrm{l}$ of epididymal fluid may be easily collected with this technique within 10-20 min. The micropipettes are flushed with a small amount of HTF or another sperm transport solution into a collection container by connecting a 1-ml syringe to the pipette with medical grade silicone tubing (or tubing from a butterfly needle). If glass micropipettes are not available, epididymal fluid may alternatively be collected by gentle aspiration using a $1-\mathrm{ml}$ syringe that has been prefilled with a small volume of sperm transport fluid and attached to a 25-gauge angiocatheter. The epididymal tubule is sealed with bipolar electrocautery and hemostasis is ensured. The epididymis and testis are returned to their normal anatomic position within the tunica vaginalis, which is closed with an absorbable suture. The dartos and skin are then closed. Bacitracin ointment is applied to the incision. Postoperatively, a scrotal supporter provides patient comfort and minimizes scrotal edema.

\section{INTRAPROCEDURAL EVALUATION OF ASPIRATED EPIDIDYMAL FLUID}

Intraprocedural evaluation of sperm quality within extracted epididymal fluid provides critical information that directs the duration and extent of sperm retrieval procedures. The evaluation is easier when specimen contamination with red blood cells is minimized. During MESA, a clean touch-prep slide can be made by simply touching the

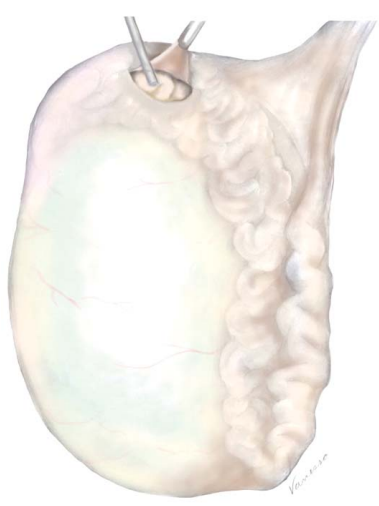

Figure 1 Schematic representation of MESA performed by aspiration of epididymal fluid by capillary action into a glass micropipette using high-power optical magnification provided by an operating microscope. MESA, microsurgical epididymal sperm aspiration. 


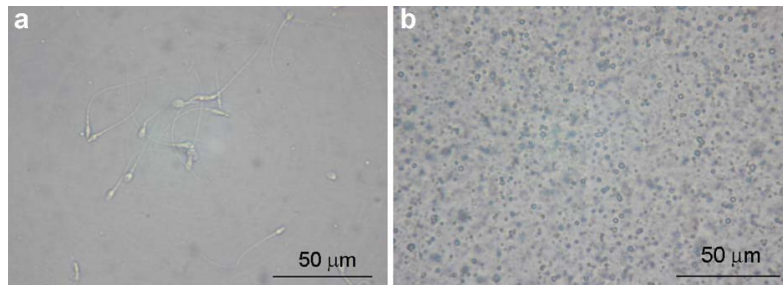

Figure 2 Example of a wet-mount slide prepared during MESA for intraoperative evaluation of sperm quality, viewed at $\times 400$ magnfication. a): Abundant, motile sperm with grossly normal morphological features. b): cellular debris and degenerated sperm heads. MESA, microsurgical epididymal sperm aspiration.

slide to the incised epididymal tubule. A droplet of HTF or another sperm transport solution is gently mixed with the epididymal fluid on the slide and the fluid mixture is covered with a cover slip. Wet mount slides can also easily be prepared from epididymal fluid after it has been collected and deposited into the sperm collection containers. Such slides may be made with more precision than touch-prep slides and are preferred for quantitative determination of sperm concentration and percent motility. Ten microliters of sperm-containing fluid is placed on a glass slide and covered with a cover slip to achieve an optimal chamber depth of approximately $20 \mu \mathrm{mol} \mathrm{L}{ }^{-1}$. Shallower chambers (less volume) for microscopic evaluation may encumber sperm motility, whereas sperm will move in and out of focus in deeper chambers (larger volume) and be more difficult to observe and count. ${ }^{29}$

Microscopic evaluation is best performed with a phase contrast microscope, but may also be performed with a standard light microscope. The wet mount slide is viewed under $\times 200-\times 400$ magnification. Standardized criteria that establish quantitative and qualitative guidelines for surgically retrieved epididymal sperm do not exist. In general, enough sperm should be harvested from every patient so that the sample may be divided into multiple aliquots that contain at least several thousand motile sperm with strictly normal morphology. This approach maximizes the opportunity for both immediate assisted reproduction and cryopreservation. The number of sample aliquots required depends upon the reproductive goals of the couple and the number of anticipated in vitro fertilization cycles that will be required to meet those goals. Example wet-mounts of aspirated epididymal fluid are seen in Figures $2 \mathbf{a}$ and $2 \mathbf{b}$.

\section{CONCLUSION}

MESA is the gold standard procedure for sperm acquisition in patients with OA of any etiology. When performed with proper technique in appropriately selected patients, this technique enables acquisition of millions of motile sperm that may be electively cryopreserved for use in multiple ICSI cycles to yield optimal reproductive outcomes.

\section{COMPETING FINANCIAL INTEREST}

The authors declare no competing financial interests.

1 Stahl PJ, Stember DS, Goldstein M. Contemporary management of male infertility. Annu Rev Med 2012; 63: 525-40.
2 Schlegel PN. Evaluation of male infertility. Minerva Ginecol 2009; 61: 261-83.

3 Stahl PJ, Stember DS, Schlegel PN. Interpretation of the semen analysis and initial male factor management. Clin Obstet Gynecol 2011; 54: 656-65.

4 Male Infertility Best Practice Policy Committee of the American Urological Association, Practice Committee of the American Society for Reproductive Medicine. Report on evaluation of the azoospermic male. Fertil Steril 2006; 86: S210-5.

5 Meng MV, Black LD, Cha I, Ljung BM, Pera RA et al. Impaired spermatogenesis in men with congenital absence of the vas deferens. Hum Reprod 2001; 16: 529-33.

6 McLachlan RI, O'Bryan MK. Clinical review\#: state of the art for genetic testing of infertile men. J Clin Endocrinol Metab 2010; 95: 1013-24.

7 Jow WW, Steckel J, Schlegel PN, Magid MS, Goldstein M. Motile sperm in human testis biopsy specimens. J Androl 1993; 14: 194-8.

8 Craft I, Tsirigotis M, Bennett V, Taranissi M, Khalifa Y et al. Percutaneous epididymal sperm aspiration and intracytoplasmic sperm injection in the management of infertility due to obstructive azoospermia. Fertil Steril 1995; 63: 1038-42.

9 Fahmy I, Kamal A, Aboulghar M, Mansour R, Serour Gl et al. Percutaneous aspiration biopsy using an intravenous catheter for testicular sperm retrieval in patients with obstructive azoospermia. Reprod Biomed Online 2004; 9: 102-5.

10 Glina S, Fragoso JB, Martins FG, Soares JB, Galuppo AG et al. Percutaneous epididymal sperm aspiration (PESA) in men with obstructive azoospermia. Int Braz J Urol 2003; 29: 141-5; discussion 145-6.

11 Hovatta O, Moilanen J, von Smitten K, Reima I. Testicular needle biopsy, open biopsy, epididymal aspiration and intracytoplasmic sperm injection in obstructive azoospermia. Hum Reprod 1995; 10: 2595-9.

12 Janzen N, Goldstein M, Schlegel PN, Palermo GD, Rosenwaks Z et al. Use of electively cryopreserved microsurgically aspirated epididymal sperm with IVF and intracytoplasmic sperm injection for obstructive azoospermia. Fertil Steril 2000, 74: 696-701.

13 Levine LA, Dimitriou RJ, Fakouri B. Testicular and epididymal percutaneous sperm aspiration in men with either obstructive or nonobstructive azoospermia. Urology 2003; 62: 328-32.

14 Lin YH, Huang LW, Seow KM, Huang SC, Hsieh ML et al. Intentional cryopreservation of epididymal spermatozoa from percutaneous aspiration for dissociated intracytoplasmic sperm injection cycles. Acta Obstet Gynecol Scand 2004; 83: 745-50.

15 Meniru GI, Gorgy A, Podsiadly BT, Craft IL. Results of percutaneous epididymal sperm aspiration and intracytoplasmic sperm injection in two major groups of patients with obstructive azoospermia. Hum Reprod 1997; 12: 2443-6.

16 Okada H, Dobashi M, Yamazaki T, Hara I, Fujisawa M et al. Conventional versus microdissection testicular sperm extraction for nonobstructive azoospermia. J Urol 2002; 168: 1063-7.

17 Rajfer J, Binder S. Use of biopty gun for transcutaneous testicular biopsies. J Uro 1989;142:1021-2.

18 Schroeder-Printzen I, Zumbe J, Bispink L, Palm S, Schneider U et al. Microsurgical epididymal sperm aspiration: aspirate analysis and straws available after cryopreservation in patients with non-reconstructable obstructive azoospermia. MESA/TESE Group Giessen. Hum Reprod 2000; 15: 2531-5.

19 Sheynkin YR, Ye Z, Menendez S, Liotta D, Veeck LL et al. Controlled comparison of percutaneous and microsurgical sperm retrieval in men with obstructive azoospermia. Hum Reprod 1998; 13: 3086-9.

20 Shrivastav P, Nadkarni P, Wensvoort S, Craft I. Percutaneous epididymal sperm aspiration for obstructive azoospermia. Hum Reprod 1994; 9: 2058-61.

21 Nicopoullos JD, Gilling-Smith C, Almeida PA, Norman-Taylor J, Grace I et al. Use of surgical sperm retrieval in azoospermic men: a meta-analysis. Fertil Steril 2004; 82: 691-701.

22 van Peperstraten A, Proctor ML, Johnson NP, Philipson G. Techniques for surgical retrieval of sperm prior to intra-cytoplasmic sperm injection (ICSI) for azoospermia. Cochrane Database Syst Rev 2008; (2): CD002807.

23 Buffat C, Patrat C, Merlet F, Guibert J, Epelboin S et al. ICSI outcomes in obstructive azoospermia: influence of the origin of surgically retrieved spermatozoa and the cause of obstruction. Hum Reprod 2006; 21: 1018-24.

24 Anger JT, Wang GJ, Boorjian SA, Goldstein M. Sperm cryopreservation and in vitro fertilization/intracytoplasmic sperm injection in men with congenital bilateral absence of the vas deferens: a success story. Fertil Steril 2004; 82: 1452-4.

25 Cayan S, Lee D, Conaghan J, Givens CA, Ryan IP et al. A comparison of ICSI outcomes with fresh and cryopreserved epididymal spermatozoa from the same couples. Hum Reprod 2001; 16: 495-9.

26 Bachtell NE, Conaghan J, Turek PJ. The relative viability of human spermatozoa from the vas deferens, epididymis and testis before and after cryopreservation. Hum Reprod 1999; 14: 3048-51.

27 Matthews GJ, Goldstein M. A simplified method of epididymal sperm aspiration. Urology 1996; 47: 123-5.

28 Zenke U, Jalalian L, Shen S, Turek PJ. The difficult MESA: findings from tubuli rect sperm aspiration. J Assist Reprod Genet 2004; 21: 31-5.

29 World Health Organization. WHO Laboratory Manual for the Examination and Processing of Human Semen. 5th ed. Geneva: World Health Organization; 2010. 